\title{
RELIGIÓN, POLÍTICA Y SOCIEDAD: LOS PRODIGIA EN LA ROMA REPUBLICANA
}

\author{
Religion, Politics and Society: Roman Republican Prodigies
}

\author{
José Manuel ALDEA CELADA* \\ Universidad de Salamanca \\ E-mail: jmaldea@usal.es
}

Fecha de recepción: 21-01-2010

Fecha de aceptación: 05-02-2010

\begin{abstract}
RESUMEN: En esta comunicación se pretende hacer un estudio sistemático de las fuentes literarias y bibliográficas que nos relatan o enumeran los prodigia para extraerlos y elaborar una base de datos. A partir de su elaboración, se hará un análisis estadístico que nos permita ver hasta qué punto la metodología estadística es aplicable y útil para la Historia Antigua, especialmente en la Roma republicana. Con los resultados del análisis estadístico se pretenderá aclarar, si es realmente posible, aspectos relativos a la posible implicación política de los prodigia, el papel de los sacerdocios públicos ante estos eventos y a la repercusión que todo ello pudo tener en la sociedad. La información estadística obtenida se presenta de forma clara y accesible, sin perderse en cifras y fórmulas que sólo nos apartarían de la investigación que deseamos llevar a cabo.

Palabras Clave:Religión romana, análisis cuantitativo, prodigios públicos, sacerdocios públicos, adivinación, política republicana, metodología.

ABSTRACT: The final objective of this study is to make an rapprochement between Ancient History and the quantitative analysis. First of all, we have studied the literary and bibliographic sources that relate or enumerate the prodigia to create a database with them. After that, we started our quantitative or statistical analysis. With the results of this analysis, we wondered if the statistical methodology could be useful to the studies on Ancient History, specially in relation with the Roman Republic. Moreover, we used this analysis to rese-
\end{abstract}


arch many aspects related to the Roman Republic religion, like the interference between the politics and the prodigies, the role played by the public priesthoods and the possible consequences in the Roman society. The statistical information obtained is presented in a clear and accessible way.

Keywords: Roman religion, Quantitative analysis, Public prodigies, Public priestshoods, Divination, Republican politics, Methodology.

\section{INTRODUCCIÓN:}

"Maiores statas sollemnesque caeremonias pontificum scientia, bene gerendarum rerum auctoritates augurum obseruatione, Apollinis praedictiones uatum libris, portentorum depulsiones Etrusca disciplina explicari noluerunt. prisco etiam instituto rebus dininis opera datur, cum aliquid commendandum est, precatione, cum exposcendum, uoto, cum soluendum, gratulatione, cum inquiriendum uel extis uel sortibus, impetrito, cum sollemni ritu peragendum, sacrificio, quo etiam ostentorum ac fulgurum denuntiationes procurantur" (Val. Max., 1. 1. 1-10) ${ }^{1}$.

Esta cita de Valerio Máximo nos acerca al tema que vamos a tratar en esta comunicación. Dentro de la religión romana republicana, nuestro estudio se centra en un aspecto concreto y bien delimitado, el de los prodigios públicos que acontecieron en Roma hasta la época de Augusto. El enfoque con el que pretendemos aproximarnos, parte de un análisis cuantitativo de los prodigios y de varios elementos que se relacionan directamente con ellos, como las expiaciones (supplicatio, obsecratio, etc.) y las intervenciones de los sacerdocios públicos. A partir de este análisis, nuestro objetivo es obtener unas conclusiones en términos numéricos que estudiaremos comparativamente con los resultados de las investigaciones referidas a este tema. Con ello pretendemos saber hasta que punto son representativos y útiles los datos cuantitativos en la historia antigua.

En primer lugar, haremos un obligado recorrido por las fuentes literarias relativas a nuestro estudio. Sin duda, debemos comenzar con la obra de Tito Livio, Ab urbe condita libri, ya que es fundamental. Tito Livio recoge, casi año por año, los prodigios que fueron comunicados al senado e incluso las expiaciones que fueron recomendadas por los sacerdotes competentes, lo que lo convierte en la fuente más completa a la hora de aportarnos los datos para la cuantificación. El problema que se nos presenta es que la obra no se conserva en su totalidad, haciendo que el número de datos esté de entrada condicionado. El hecho de que Tito Livio recoja estos datos con tanto interés, puede deberse al valor que la propia sociedad romana les daba, pues, y como nos señala él mismo, el senado se ocupaba de los prodigios incluso antes que de los asuntos bélicos: "perpetratis quae ad pacem deum pertinebant, de re publica belloque gerendo et quantum copiarum et ubi quaeque essent consules ad senatum rettulerunt" (Liv., 24. 11. 1) ${ }^{2}$. Además, debemos tener en cuenta que utilizaba como fuentes los Libripontificales 
y los Annales maximi, donde se recogían todos los prodigios año por año.

En una línea parecida a la de la obra de Livio, se encuentra el Liberprodigiorum de Julio Obsecuente. A diferencia de Livio, Obsecuente hace una mera relación de los prodigios, sin especificar casi nunca ni a quien se le encarga la expiación ni en que consiste ésta, tal como debería aparecer en los Annales maximi. A pesar de esto, no deja de ser una obra de gran importancia para completar el vacío de información dejado por Livio, especialmente en el período comprendido entre el 166 y el 99 a. C., ya que en ocasiones es la única referencia conservada.

Sin duda, las anteriores son las fuentes principales, pero son otros muchos los autores clásicos que dedicaron parte de su obra al comentario de los prodigios. Uno de ellos es Dionisio de Halicarnaso (Historia antigua de Roma), que suele recoger prodigios que tienen una importancia especial para la historia religiosa e incluso política de Roma, como el incesto de las vestales o la introducción de las divinidades extranjeras. Algo similar es lo que ocurre con Dión Casio (Historia romana). Volviendo a autores romanos, tenemos que volver a hablar de Valerio Máximo (Facta et dicta memorabilla), pues dedica por completo su primer libro a temas relacionados con la religión romana, incluidos los prodigios. Las Quaestiones Romanae de Plutarco son otra fuente, aunque la relación de prodigios es muy escasa.

De igual modo, hemos de hacer una referencia a los autores de la Antigüedad tardía, ya que no son pocos los prodigios y expiaciones que recogen, aunque su perspectiva e intención son muy diferentes a las de los autores anteriores. En este caso, lo que pretenden es hacer una crítica a la religión pagana y a la adivinación. Entre los autores que podemos mencionar se encuentran Orosio (Aduersus paganus historiarum libri), Agustín de Hipona ( $D e$ cinitate Dei) y Lactancio (Dininarum institutiorum libri).

Hemos dejado pretendidamente para el final la figura de Cicerón, dado que se trata de una fuente compleja. Es cierto que Cicerón no escribió ninguna obra que nos permita obtener datos cuantitativamente relevantes sobre los prodigios, pero escribió obras fundamentales relacionadas con la interpretación de los prodigios y la adivinación romana, a saber, Pro domo sua, De haruspicum responso y De dininatione. A partir de estas obras se ha escrito mucho sobre si los romanos creían o no en los prodigios, sobre lo que creía el propio Cicerón (y en ambos casos nos referimos a "creer" en términos de fe religiosa) y sobre la hipocresía que algunos pretenden ver en los prodigios y sus expiaciones. Pero, a este respecto, compartimos la idea de Rasmussen de que "the institution of Roman public portents revolves far more around social, religious, and political behaviour then around faith's , los prodigios son un elemento más de la religio romana y, por tanto, tienen muy bien definido su tratamiento por parte de los diferentes elementos del Estado (tanto del senado como de los sacerdocios públicos). 


\section{Metodología:}

Podemos definir prodigio "as any unsual occurance in society which is reported to the senate and accepted by that body as a prodigium publicum requiring ritual expiation" ", tal como lo hace Rasmussen. Al compartir esta definición, dejamos conscientemente fuera de esta investigación los escasos ejemplos de prodigios considerados privados, ya que el senado tampoco los tenía en cuenta (Liv., 43. 13. 5). En la obtención de los datos hemos seguido unos criterios de selección concretos, uno de ellos lo acabamos de citar. Dentro de los prodigios que el senado consideró de interés público, nosotros hemos seleccionado exclusivamente los que tuvieron lugar en la ciudad de Roma y el ager Romanus. Y de estos, hemos estudiado los que tuvieron lugar entre el 504 y el 16 a. C., es decir, durante todo el período republicano y en tiempos de Augusto.

Para la creación de la base de datos han sido fundamentales tres obras que contenían tablas recogiendo prodigios, aunque todas ellas con otros criterios de selección diferentes a los que hemos planteado ${ }^{5}$. Con estos criterios de selección obtuvimos un total de 334 prodigios, una cifra elevada, aunque tratándose de casi 500 años, cabe preguntarse hasta qué punto los resultados pueden ser representativos. De hecho, si esta cifra puede hacernos dudar de la valía de las conclusiones que vamos a obtener, todavía resulta más difícil analizar las intervenciones sacerdotales y las expiaciones, debido a que su número es todavía más reducido (102 intervenciones sacerdotales y 202 expiaciones).

Terminada la elaboración de la base de datos, sin duda la parte más costosa y lenta de la investigación, el siguiente paso era más sencillo, simplemente había que convertir en datos numéricos los prodigios, las intervenciones sacerdotales y las expiaciones para luego agruparlos por categorías que nos permitieran conocer los porcentajes relativos a su mayor o menor aparición, concentración en intervalos cronológicos, etc.

El primer punto consistió en crear una tabla con los prodigios más frecuentes, para lo que incluimos a todos aquellos prodigios que se hubieran repetido al menos dos veces. De este modo, el resultado fueron 274 prodigios, los que constan en la gráfica 1, agrupados en 33 categorías y siendo el rayo el más repetido, con un total de 44 . A continuación vamos a explicar el contenido de cada una de las categorías. En rayo se incluyen todos los prodigios relacionados con este fenómeno natural, de igual modo, lo hacemos con tormenta, terremoto, incendio, trueno, crecida y arcoiris; en lluvia se agrupan todos los tipos de lluvias anómalas, como lluvia de rocas, de sangre, de leche, etc.; en plaga, las plagas, pestes y epidemias; en búbo, el avistamiento de este ave en la ciudad de Roma, lo mismo para lobo, abeja, serpiente, cuervo y buitre; en cielo se incluyen fenómenos extraños avistados en el cielo, como luces, an- 
torchas, etc.; en nace, los nacimientos de animales y personas con alguna peculiaridad, salvo los hermafroditas, que tienen una categoría propia; con habla hacemos alusión a cuando animales (generalmente bueyes) o niños muy pequeños hablaron; en estatua se han incluido los prodigios en los que intervienen estatuas, como las que se giran por sí mismas; en fluye, las referencias a riachuelos o hilos de sangre y otros tipos que surgieron en diferentes lugares de la ciudad; lanza se refiere principalmente a cuando se movieron de forma involuntaria las lanzas de Marte en la Regia; en sol están los fenómenos como el avistamiento de dos o más soles, pero los eclipses tienen una categoría propia; en visión recogemos las visiones peculiares notificadas al senado, como una lucha de armas, gente togada sin que realmente hubiera nadie, etc.; en suda, los prodigios en los que varias estatuas sudaron; con voz nos referimos a cuando se escucharon voces sin saber de donde ni quien provenían, lo mismo ocurre con aúlla, tratándose en este caso de aullidos de perros o lobos generalmente; el incesto de las vestales se recoge en la categoría vestal; con hígado englobamos los casos en los que al examinar el hígado de una víctima en un sacrificio no aparecía la protuberancia; en mula, cuando una mula daba a luz; si las vestales dejaban que la llama de Vesta se apagara, era un prodigio, recogido en llama; por último, con escudo agrupamos los dos casos en que los escudos sagrados se movieron y aparecieron con manchas de sangre.

La gráfica 2 es el resultado del recuento de las intervenciones llevadas a cabo por los colegios sacerdotales públicos. Son siete las categorías empleadas, Xuiri s. f., que abarca las intervenciones del colegio de los decemuiri sacris faciundis, incluidas las de cuando eran duumuiri (hasta el 367, año en el que se amplía de dos a diez miembros, ver Liv., 6. 37.12) y quindecemuiri (a partir de Sila). Cuando en las fuentes había una referencia explícita a la consulta de los libros sibilinos, lo hemos considerado como una intervención más de los Xuiri, mientras que cuando conocíamos la expiación y ésta solía ser de las recomendadas por los Xuiri, si no eran citados, por prudencia, no lo hemos considerado como intervención suya. La segunda categoría es la de sin referencia, en la que se cuentan los años en los que conocemos los prodigios y la expiación pero no la intervención de los sacerdotes. La tercera es haruspices, que, al igual que con los Xuiri, no se les han asignado intervenciones que por sus características podrían corresponderles ${ }^{6}$. Los pontifices son la cuarta categoría y en este caso hemos hecho una excepción, ya que les hemos atribuido la intervención en todos los casos en los que una vestal rompía su voto de castidad, pues son el colegio encargado de su control y protección (ver Liv., 22. 57. 1-3 para el caso de las vestales Opimia y Floronia en el 216, en el que sí se menciona la intervención de los pontifices). Las tres categorías restantes no han presentado ningún problema, lo que en parte puede ser debido a que su intervención fue limitada. Se trata de mánteis, término griego que aparece así mencionado en Dionisio de Halicarnaso (ver Dion. Hal., 8. 89. 3-5 y 10. 2. 2-6, por ejemplo), de senado, que podía establecer la expiación directamente 
sin consultar a los colegios sacerdotales (ver Liv., 3. 7. 6-8 y 10. 23. 1, por ejemplo), y de uates, término latino que se podría corresponder a adivino (ver Liv., 2. 42.10 y 7. 6. 3).

La gráfica 3 siguió un proceso de creación similar al de la gráfica 1. En este caso se recogen las expiaciones de las que tenemos referencias, reflejándose específicamente las que se repitieron por lo menos dos veces y habiendo una categoría en la que se sitúan las expiaciones que no se repitieron, un 13\% del total. De las 22 expiaciones que se repitieron, la más frecuente es la supplicatio, con 36 referencias.

En último lugar está la gráfica 4, en la que podemos ver el número de prodigios agrupados por intervalos temporales. El primer intervalo es el comprendido entre el 504, fecha de la que data el primer prodigio sucedido en la Roma republicana, hasta el 264, momento en el que da comienzo la primera Guerra Púnica; el segundo comprende la primera Guerra Púnica (del 264 al 241); el tercero, el período hasta que comienza la segunda Guerra Púnica (del 241 al 218); el cuarto, la segunda Guerra Púnica (218 al 201); el quinto, el período de entreguerras, hasta la tercera Guerra Púnica (del 201 al 149); el sexto, la tercera Guerra Púnica (del 149 al 146); el séptimo, del final de esta guerra hasta el final de la primera Guerra Civil (del 146 al 84); el octavo, desde que concluye esta guerra hasta la muerte de César (del 84 al 44); por último, de la muerte de César al último prodigio que tuvo lugar en Roma en tiempos de Augusto (del 44 al 16). La elección de estos intervalos no es casual, ya que con ellos pretendemos ver si siguen los dos parámetros que la historiografía viene defendiendo tradicionalmente. El primero dice que los prodigios responden a una situación de tensión social e incluso de histeria colectiva ante una coyuntura adversa (guerras púnicas y guerras civiles), teoría defendida a partir de fragmentos de Tito Livio como este: "Romae ad primum nuntium cladis eius cum ingenti terrore ac tumultu concursus in forum populi factus est' (Liv., 22. 7. 6). El segundo los considera un instrumento político, especialmente a partir de algunos casos concretos que tuvieron lugar en los últimos años de la República (ver, por ejemplo, Obseq., 46). Al analizar los resultados intentaremos ver hasta qué punto se adaptan a estas dos teorías o si por el contrario cabe pensar en otra interpretación posible.

\section{ANÁLISIS DE LOS RESULTADOS OBTENIDOS:}

Una vez terminada la organización y puesta a punto de los datos recogidos, el siguiente paso es analizar los resultados e interpretarlos. Comenzaremos volviendo a hacer especial hincapié en la importancia que tenían los prodigios y sus expiaciones. El hecho de que el senado lo considera una prioridad ya nos lo remarca, pero también el hecho de que conservemos refe- 
rencias de prodigios para casi cuatrocientos años de la historia republicana. De no ser una cuestión de cierta relevancia en la sociedad romana, ¿qué sentido tendría que los historiadores de la época se dedicaran a recogerlos en sus obras? Está claro que los prodigios son un elemento más dentro de la religión oficial en Roma y con un papel fundamental para el mantenimiento de la pax deorum. La ruptura de la pax deorum era manifestada por parte de los dioses, como dice Monaca, "gli dèi mandano agli nomini di continuo segnali della loro presenza e della loro volontà ed il mondo è lo scenario dei loro interventi" ". Y esa voluntad de los dioses debía ser interpretada para poder restaurar la pax deorum.

Esto nos lleva a centrarnos en el estudio de las intervenciones sacerdotales. Los sacerdocios públicos, como ya hemos indicado, eran los encargados, a petición del senado, de recomendar las expiaciones. Esta labor corrió a cargo, en la mayoría de ocasiones, de los Xuiri sacris faciundis y de los haruspices, correspondiéndoles el 35 y el 16\% de las intervenciones respectivamente. El caso de los Xuiri es especialmente interesante, ya que son un colegio propiamente romano constituido, según la tradición, tras la expulsión de los reyes y cuya tarea fundamental era consultar los libros sibilinos siempre que el senado se lo encomendara (Dion. Hal., 4. 62). También eran competencias de los Xuiri la vigilancia de los cultos extranjeros y la supervisión de las actividades religiosas realizadas según el llamado ritus graecus, de entre las que debemos destacar los lectisternia, ocho según la gráfica 3. Los lectisternia nos permiten analizar la evolución de la religión romana hacia una cada vez más clara helenización ${ }^{8}$. Esta helenización se puede ver en los dioses que participan en los lectisternia, tanto por su número como por sus empajeramientos. Mientras que en el primero (celebrado en el 399, Liv. 5. 3. 5-6) se encontraban Apolo y Latona, Hércules y Diana y Mercurio y Neptuno, en el del 217 encontramos ya a los doce dioses principales, Júpiter y Juno, Neptuno y Minerva, Marte y Venus, Apolo y Diana, Vulcano y Vesta y Mercurio y Ceres (Liv., 22. 10. 9-10). La presencia de Vulcano y Vesta se puede vincular con el peso de las divinidades romanas tradicionales. No nos detendremos en la naturaleza de los libros sibilinos ${ }^{9}$, simplemente señalar que se integran dentro de la tradición helénica de textos oraculares y que, en palabras de Bouch-Leclerq, "Rome se trouvait maintenant dans la situation de la plupart des villes grecques, qui tenaient sous clef dans leur acropole des collections d'oracles et autres documents secrets, ou des villes étrusques qui avaient leurs libri fatales" ${ }^{10}$. Los Xuiri recurrían a ellos en busca de soluciones de carácter ritual y no de predicciones de futuro, lo que puede explicar que sea común encontrar expiaciones estandarizadas, como la supplicatio, la obsecratio, la lustratio y el lectisternium, a parte de los sacrificios de hostiae maiores y lactentes. Este tipo de expiaciones, como podemos ver en la gráfica 3, suman 99 (el 44\%), casi la mitad. 
El caso de los haruspices es bien diferente, pues son un colegio extranjero y siempre será tenido en cuenta como tal. Los haruspices eran conocedores de la Etrusca disciplina, citada por Valerio Máximo (uide supra) y con unas funciones bastante delimitadas. Los romanos contaron ellos para un tipo de prodigios más o menos concretos, como los rayos, truenos y relámpagos y el descubrimiento de hermafroditas. La llegada de los haruspices a Roma no está del todo clara, pero lo más probable es que tuviera lugar en la época de los Tarquinios ${ }^{11}$. La intervención de este colegio también dependía del senado, que en ocasiones pedía una interpretación tanto a los Xuiri como a los haruspices, tal como sucedió en el 172, cuando un rayo destruyó la columna rostrata del Capitolio (Liv., 42. 20. 1-6). Sobre el arte fulgural, podemos citar la síntesis que North hace a partir de varios fragmentos de De haruspicum responso de Cicerón, "it identifies the gods to whom ritual action is owed; it explains what the significance of the prodigy is in terms of human errors and religious crimes; and it interprets the prodigy as a set of warnings about the future, admittedly unspecific but definitely predictive in form" ${ }^{\prime 2}$. La presencia de los haruspices se puede rastrear tras la mayoría de los casos de aparición de un hermafrodita y la expiación que recomiendan está siempre en la misma línea, los monstra deben desaparecer del mundo humano, por lo que los arrojan vivos al mar o los queman vivos (ver, por ejemplo, Obseq., 24; 34 y 48).

El papel de los pontifices es mucho más limitado, con solamente el 9\% de las intervenciones. Cabe destacar el papel que debieron jugar en relación con los crimina incesti de las vestales, considerados prodigios públicos. Por "los riesgos para la comunidad que implicaba no respetar el voto de castidad por parte de estas sacerdotisas, era necesaria la imposición de un cruel y ejemplarizante castigo, el de ser enterradas vivas" ${ }^{\prime 13}$. Serían los pontifices los encargados de controlar a las vestales y de hacer cumplir este castigo.

El castigo que se imponía a las vestales nos lleva a comentar a continuación una de las expiaciones más interesantes por su significación histórica y peculiaridad, ya que numéricamente tiene escasa representatividad. Se trata del enterramiento de personas vivas, el único ejemplo de sacrificio humano en Roma. Siguiendo los datos de la gráfica 3, son un total de seis casos, que pueden dividirse en dos categorías, por un lado los enterramientos de vestales vivas (tres, en el 472, el 337 y el 216) y por otro el enterramiento de una pareja de griegos y una pareja de galos (otros tres, en el 225, el 216 y el 114). La consulta de los libros sibilinos está detrás de todos los casos de los enterramientos de griegos y galos, de hecho, eran los Xuiri los encargados de llevar a cabo el ritual ${ }^{14}$. Realmente, este tipo de expiación presenta problemas a la hora de interpretarla, pues ni sus orígenes ni su justificación son fácilmente discernibles a partir del estudio de las fuentes que lo citan. Siguiendo a Bémont, "nous devons donc nous borner à supposer que l'inadéquation du sacrifice à la situation militaire est due à l'utilisation d'un rituel ancien, probablement étrusque, dont 
on a pu, peut-être, respecter la stipulation à la lettre, à la faveur, faute de menace grecque précise, d'un peril gaulois très réep' 15 . Esta interpretación se basa en que enterrar a un griego y un galo no tendría justificación militar, ya que en el contexto de las guerras púnicas habría tenido más sentido que los sacrificados fueran cartagineses. Podemos afirmar, por tanto, que estas expiaciones tienen un gran valor a nivel histórico, aunque si nos hubiéramos limitado a dar importancia a las más representativas numéricamente, habrían quedado, sin duda, fuera de la investigación. En cambio, nos hubiéramos centrado en otras expiaciones, como las supplicationes, que tienen exclusivamente un papel ritual muy estandarizado y una trascendencia en términos históricos mucho menor.

No podemos dejar de mencionar que la naturaleza de los prodigios también cambia con el tiempo. Si bien es cierto que hay algunos que son una constante en todo el período que hemos estudiado, por el contrario, hay algunos que son más definitorios de un momento concreto. Esto tiene lugar especialmente en los últimos años de la República, desde la muerte de César al 16 (año del último prodigio que hemos incluido en la base de datos). Podemos citar algunos ejemplos, en el año 44, durante los juegos de Venus Genetrix se ve una estrella con una estela larga; una estrella brilla en el cielo durante siete días; la mujer de César tiene un sueño y Octavio, al entrar en Roma, parece rodeado por el final de un arcoiris (ver Obseq., 67-68). Parece que los prodigios toman una dimensión política, y están encaminados a favorecer la divinización de César y la llegada al poder de Augusto. Aunque no aparece en nuestra lista, es en estas fechas cuando se crearía el mito según el cual Augusto es hijo de Apolo (para el texto completo ver Suetonio, Diu. Aug., 94 y Dio., 45. 1. 2). A través de un prodigio, explicarían el origen divino de Augusto, cuya madre, Atia, sería engendrada por Apolo convertido en serpiente mientras ésta participaba en unos rituales nocturnos en el templo del dios en compañía de otras matronas romanas. Según Wildfang, se trataría de una construcción basada en modelos orientales, incluso apunta que "these dreams' symbolism was directed to an eastern audience" y que "their message matched Augustus' propaganda in that region of the empire in the ten years between the peace of Brindisium and Antony's death"16. Estamos ante una clara transformación del prodigio, que, en casos puntuales, sobrepasa los límites de la religio pública para favorecer unos intereses privados.

A continuación, vamos a centrarnos en la gráfica 4 y de este modo volver sobre un tema que esbozamos en el apartado de metodología. Como podemos observar, el período que mayor porcentaje de prodigios presenta es el del 146 al 84, con un 31\%, seguido, ambos con el 16\%, por el del 201 al 149 y el del 84 al 44 . Viendo estos resultados, en principio sí podría decirse que las dos tendencias tradicionales vendrían a confirmarse, pues en el primer intervalo la mayor concentración se produce a partir del 103-104, aproximadamente, coincidiendo con un momento de agitación política y social. De 
hecho, el ejemplo que sugeríamos anteriormente, en Obseq. 46, corresponde a este intervalo. También está a favor de las tesis tradicionales el hecho de que durante la segunda Guerra Púnica, un período de sólo 17 años, tenga el $11 \%$ de los prodigios. Pero lo que nos interesa saber realmente es si se trata de una consecuencia política y social. En lo referente a lo social, ver los prodigios como una superstitio favorecida por las masas temerosas, es, a nuestro parecer, un equívoco, ya que los prodigios fueron siempre parte de la religión pública, con unas normas precisas para su aceptación y posterior expiación. Es más, el senado decidía si hacer públicos o no los prodigios y sus expiaciones, por lo que el pueblo podría no haber estado al tanto de todos los prodigios que eran notificados al senado ni de si se prescribían determinadas expiaciones (un ejemplo en el cual el senado decide hacerlo público lo tenemos en Liv., 40. 19. 5-6: "eorum decreto (de los Xuir) supplicatio circa omnia puluinaria Romae in diem unum indicta est. isdem auctoribus et senatus censuit et consules edixerunt ut per totam Italiam triduum supplicatio et feriae essent'). Por ello, una situación convulsa puede hacer que sean notificados al senado más prodigios, pero no que éste cambie su actitud ante ellos y acceda a expiarlos todos por el simple hecho de estar en guerra o una situación similar. Durante la segunda Guerra Púnica tuvieron lugar prodigios como lluvias de piedra y sangre, estatuas que sudan, el incesto de las vestales Opimia y Floronia, visiones, rayos, etc. (Liv., 21. 46. 1-2; 21. 62; 22. 1. 8-12; 22. 36. 6-7; 22. 57.1-4), pero no son prodigios que no se documenten durante cualquiera de los otros intervalos de tiempo. Lo único que está a favor de esa teoría es la concentración de prodigios en ese breve intervalo de tiempo.

Pasemos ahora a la cuestión política. Ya hemos apuntado que a finales de la República se produce un cambio en la naturaleza de los prodigios, pero es necesario matizar esta idea. No hay duda del cambio que se está produciendo, lo que debemos tener en cuenta es si el número de este tipo de prodigios es el suficiente como para considerarlo una tendencia general o si, por el contrario, se trataría de un grupo de prodigios restringidos a un grupo político y social concreto. Analizando los prodigios que tuvieron lugar desde el 103-104, vemos que la gran mayoría son prodigios que podríamos calificar de "comunes", pudiendo considerarse de valor político los siguientes: dos cuervos se pelean sobre una asamblea en la que se discute una distribución de tierras y la ley no se aprueba por recomendación de los haruspices (ver Obseq. 46 y Aul. Gel., N. A., 4. 6. 1-2); en el 87 se producen prodigios en el campamento de Pompeyo y su propia muerte y en esa fecha se publica el oráculo sibilino sobre Cinna (ver Obseq., 56a; Plin., N. H., 2. 92; Oros., 5. 19. 18); los angures posponen los comitia tras alcanzar un rayo los templos de Luna y Ceres (ver Apian., B. C., 1. 78); en el 56 se publica el oráculo sibilino sobre Ptolomeo (ver Dio., 39. 15-16); en el 54 se publica el oráculo sibilino sobre Gabinio (ver Dio., 39. 60 y 61); en el 43 hay siete prodigios relacionados con Pansa y con Octavio (ver Obseq., 69; Dio., 46. 33); a estos habría que 
añadir los mencionados más arriba en relación con César y el sueño sobre el origen divino de Augusto. Esta lista de prodigios sólo supone unos veinte de los 151 que tuvieron lugar desde el 103-104 hasta el 16, por lo que sería imprudente afirmar que los prodigios servían de forma clara a los intereses políticos. Del mismo modo, compartimos la idea de Rasmussen de que la utilización política de unos pocos prodigios a finales de la República no implica la decadencia ni de la adivinación romana ni de la religión ${ }^{17}$.

\section{CONCLUSIONES:}

Si comenzamos con una cita de Valerio Máximo, concluiremos citando a Cicerón: "non enim illa, quae futura sunt, subito exsistunt, sed est quasi rudentis explicatio sic traductio temporis nibil noui efficientis et primum quidque replicantis. quod et ei vident, quibus naturalis dininatio data est, et ei, quibus cursus rerum obseruando notatus est. qui etsi causas ipsas non cernunt, signa tamen causarum et notas cernunt; ad quas adhibita memoria et diligentia et monumentis superiorum efficitur ea divinatio, quae artificiosa dicitur, extorum, fulgorum, ostentorum signorumque caelestium" (Cic., De diu., 1. 127) ${ }^{18}$. Con estas líneas, Cicerón establece una doble metodología adivinatoria, la intuitiva y la inductiva. En esta investigación hemos manejado conjuntamente ambos modelos. La adivinación intuitiva sería aquella que, siguiendo a Bouché-Leclerq ${ }^{19}$, pondría en contacto directo el alma con los dioses. La adivinación inductiva, en cambio, "n'est pas le produit direct de l'inspiration surnaturelle, mais une induction bypothétique ou conjecturale fondée sur des faits connus par l'observation. C'est l'interpretation des signes extérieurs qui recèlent la pensée

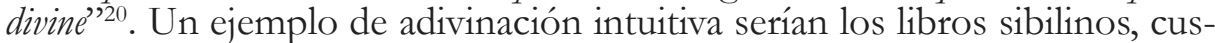
todiados por los Xuiri y de gran importancia para la ciudad de Roma, ya que habrían sido inspirados directamente a la Sibila por la divinidad. La adivinación inductiva sería la que practicaban los haruspices, el otro gran colegio sacerdotal de origen etrusco, que estaba especializado en unos prodigios determinados, como los rayos (los "fulgorum" de la cita).

Con el análisis cuantitativo hemos querido mostrar como el colegio de los Xuiri era el que tenía un papel más activo en relación a los prodigios, tanto al consultar los libros sibilinos como al proponer expiaciones sin su consulta. De la actividad de este colegio se derivaron expiaciones de tanta importancia como la introducción de cultos nuevos (posiblemente de Apolo, de Ceres, de Esculapio, de Venus Erycina, de la Magna Mater, de Ceres, de Flora, de Mens y de Venus Verticordia ${ }^{21}$ ). Con ello no pretendemos restar importancia al resto de colegios que intervenían en cuestiones de adivinación y prodigios. Es innegable que los haruspices tenían un peso específico dentro de la religión romana, pues al practicar una doctrina extranjera (Etrusca disciplina) podrían haber tenido límites y reticencias por parte de los otros colegios. 
También hemos pretendido hacer ver que la cuantificación puede ser una trampa al querer hacer el dato más o menos importante el que tiene un mayor o menor número o porcentaje. Como se ha indicado, hay prodigios y expiaciones que no fueron muy frecuentes pero que nos aportan gran cantidad de información histórica a nivel político, social y religioso. Un buen ejemplo serían las inhumanciones de personas vivas en la propia Roma y los crimina incesti de las vestales. La información cuantitativa tampoco distingue entre contextos culturales e históricos, mientras que al afrontar la historia de la religión romana desde una perspectiva social, es algo que debemos marcar y diferenciar para no caer en valoraciones anacrónicas. Por emplear el mismo ejemplo, no podríamos valorar e interpretar las inhumaciones de personas vivas de igual modo en un contexto de las guerras púnicas y en el siglo final de la República.

Por último, hemos delimitado nuestro concepto de prodigium publicum al situarlo dentro de la religio, de la religión romana pública y organizada por el Estado. No lo consideramos parte de la superstitio, como podría pensarse al estudiarlos como una consecuencia de la coyuntura bélica o socialmente inestable (el terror del pueblo que se quiere ver en citas de Tito Livio). Tampoco creemos que se trate de instrumento político, aunque sí que hemos señalado casos concretos en los que pudieron emplearse como tal. No hemos querido dejar de mencionar el cambio en la naturaleza de determinados prodigios que tuvo lugar a finales de la República, siendo para nosotros el de mayor importancia la construcción mítica del origen divino de Augusto (nacido de Apolo y Atia).

\section{Notas}

*Becario FPI de la Junta de Castilla y León, adscrito a la Universidad de Salamanca.

${ }^{1}$ En la edición: BRISCOE, John (ed.), Facta et dicta memorabilla. Valeri Maximi. 2 vols. Stutgardiae, Teubneri, 1998.

${ }^{2}$ En la edición: FOSTER, B. O. (trad.), Ab urbe condita. Liyy. Cambridge, Harvard University Press, 1967 y ss.

${ }^{3}$ RASMUSSEN, Susanne William, Public Portents in Republican Rome. Roma, L'Erma de Bretschneider, 2003, p. 195.

${ }^{4}$ RASMUSSEN, Susanne William, “Cicero's Stand on Prodigies. A Non-existent Dilemma?" en WILDFANG, Robin Lorsch y Jacob ISAGER, Divination and Portents in the Roman World. Odense, Odense University Press, 2000, p. 11.

${ }^{5}$ Se pueden ver las tablas en MacBAIN, Bruce, Prodigy and Expiation: a Study in Religion and Politics in Republican Rome. Bruselas, Latomus, 1982, pp. 82-106; ORLIN, Eric M., Temples, Religion and Politics in the Roman Republic. Leiden, E. J. Brill, 1997, pp. 203-207; RASMUSSEN, op. cit., pp. 53-116. 
${ }^{6}$ Hemos optado por este criterio, al contrario de lo que hace MacBain, op. cit., pp. 82 y ss., ya que intenta demostrar que las intervenciones de los haruspices fueron más frecuentes de lo que las fuentes nos indican explícitamente.

${ }^{7}$ MONACA, Mariangela, "Prodigi e profezie nel mondo romano: peculiarità della rivelazione sibillina" en SFAMENI GASPARRO, Giulia (ed.), Modi di comunicazione tra il divino e l'umano: tradizione profetiche, divinazione, astrologia e magia nel mondo mediterraneo antico: atti del II Seminario internazionale. Cosenza, Lionello Giordano, 2005, p. 314.

${ }^{8}$ Para el desarrollo de los lectisternia, ver GUILLÉN, José, Urbs Roma, vol. 3. Salamanca, Sígueme, 1977, pp. 108 y ss.

${ }^{9}$ Sobre los libros sibilinos, ver BOUCHÉ-LECLERQ, Auguste, Histoire de la divination dans l'Antiquité, vol. 4. Aalen, Scientia, 1978, pp. 288 y ss.; MONACA, Mariangela, op. cit., pp. 320 y ss.; ORLIN, Eric M., op. cit., pp. 76-115.

${ }^{10}$ BOUCHÉ-LECLERQ, Auguste, op. cit., vol. 4, p. 290.

${ }^{11}$ Cfr. MacBAIN, Bruce, op. cit., p. 46.

${ }^{12}$ NORTH, John, "Prophet ant Text in the Third Century B.C." en BISPHAM, Edward y Christopher SMITH (eds.), Religion in Archaic and Republican Rome and Italy. Evidence and Experience. Edimburgo, Edinburgh University Press, 2000, p. 94.

${ }^{13}$ CID LÓPEZ, Rosa María, "Las matronas y los prodigios. Prácticas religiosas femeninas en los márgenes de la religión romana”. Norba. Revista de Historia, 2007 (20), p. 16.

${ }^{14}$ Cfr. BÉMONT, Colette, "Les enterrés vivants du Forum Boarium". Mélanges d'Archéologie et d'Histoire, 72 (1960), p. 135.

${ }^{15}$ BÉMONT, Colette, op. cit., p. 141.

${ }^{16}$ WILDFANG, Robin Lorsch, "The Propaganda of Omens: Six Dreams Involving Augustus" en WILDFANG, Robin Lorsch y Jacob ISAGER (eds.), Divination and Portents in the Roman World. Odense, Odense University Press, 2000, p. 48.

${ }^{17}$ RASMUSSEN, Susanne William, op. cit., p. 52.

${ }^{18}$ ARMISTEAD FALCONER, William (trad.), Cicero. De Senectute. De Amicitia. De divinatione. Cambridge, Harvard University Press, 1923.

${ }^{19}$ BOUCHÉ-LECLERQ, Auguste, op. cit., vol. 1, pp. 273 y ss.

${ }^{20}$ Idem, p. 111.

${ }^{21}$ Ver ORLIN, Eric M., op. cit., pp. 97 y ss. 


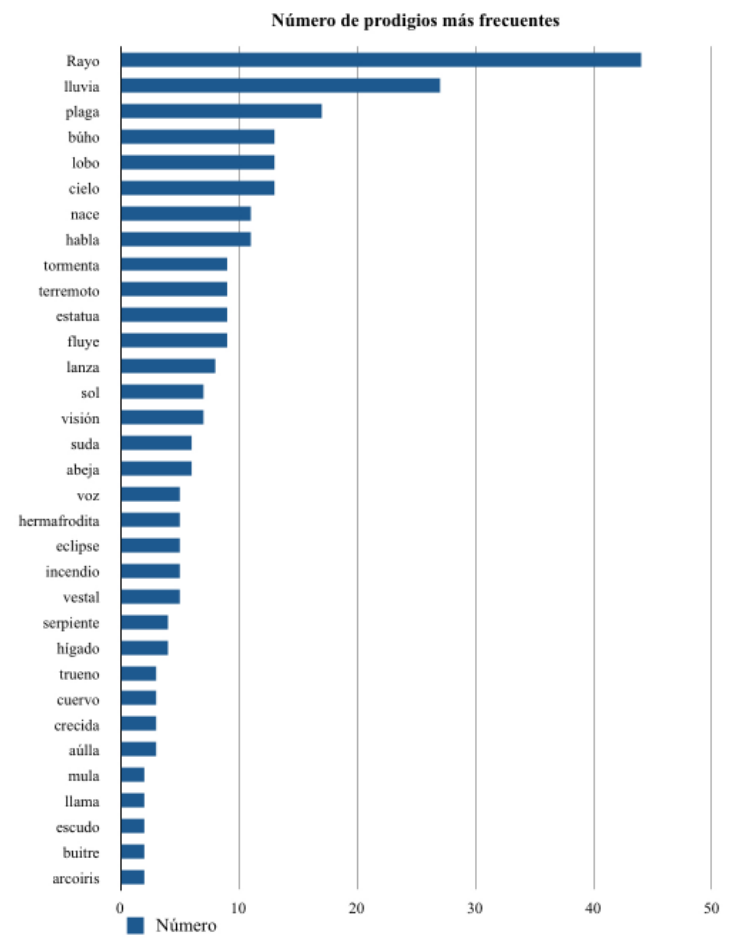

Porcentajes de las intervenciones sacerdotales

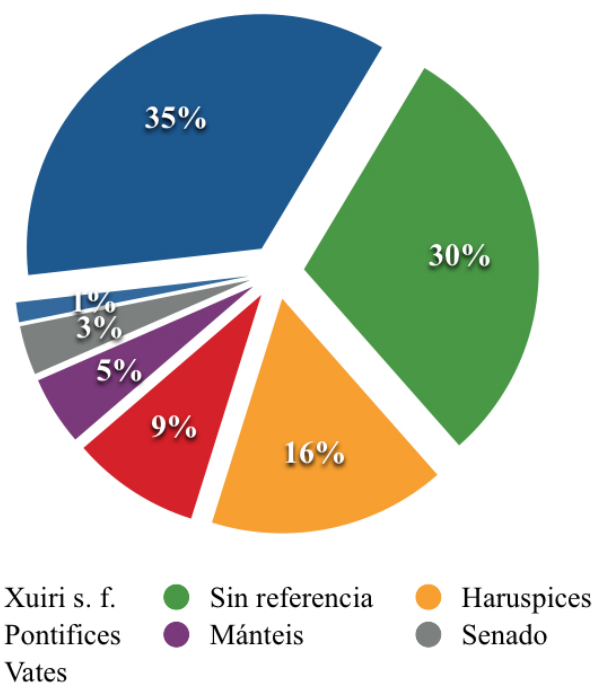




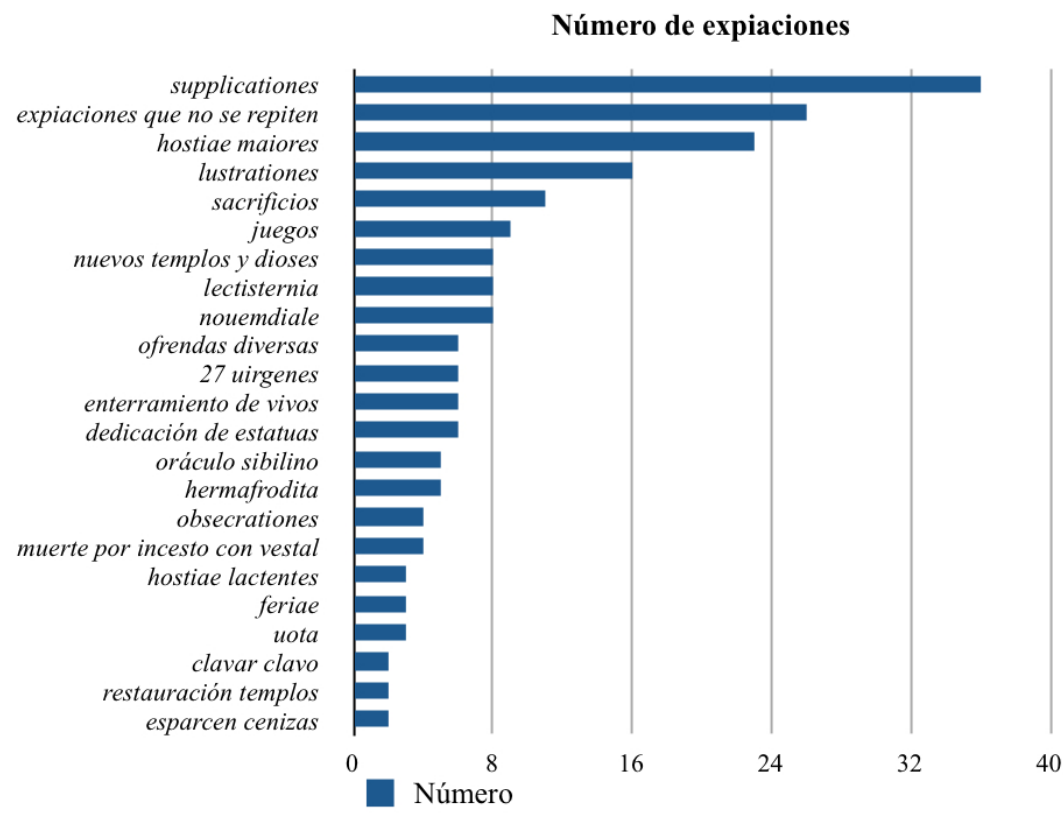

Porcentajes de prodigios por intervalos temporales
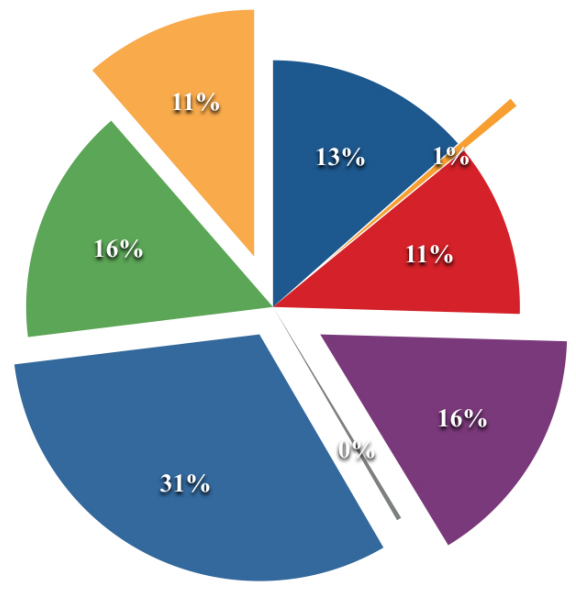

504-264

$264-241$

241-218

218-201

201-149

149-146

$146-84$

$84-44$

$44-16$ 